\title{
In memory of Professor Ali H. Nayfeh
}

\author{
Giuseppe Rega • Mohammed F. Daqaq • \\ Muhammad Hajj • Anil Bajaj
}

Published online: 16 December 2019

(C) Springer Nature B.V. 2019

Professor Ali H. Nayfeh passed away on March 27, 2017, at the age of 83. In the last 50 years, he was one of the most prominent scholars and scientists in the area of nonlinear dynamics applied to mechanics and engineering. Hundreds of students at Virginia Tech and younger colleagues all over the world were strongly influenced by his approach to treating nonlinear problems and by his scientific contributions to a great variety of fields. The journal Nonlinear Dynamics, which he timely founded in 1990, brought to a doubtless prestige, and led until the end of his life, duly dedicates this Special Issue (S.I.) to his memory. For an unintended yet subtle coincidence, the issue contains just 50 papers, by invitation only, that have undergone the regular review process. Many of Nayfeh's colleagues, mentees and students from different countries contributed to this S.I.

G. Rega ( $\square)$

Department of Structural and Geotechnical Engineering,

Sapienza University of Rome, Rome, Italy

e-mail: giuseppe.rega@uniroma1.it

M. F. Daqaq

Division of Engineering, New York University Abu Dhabi,

Abu Dhabi, UAE

e-mail: mohammed.daqaq@nyu.edu

M. Hajj

Department of Civil, Environmental \& Ocean Engineering, Stevens University of Technology, Hoboken, NJ, USA

e-mail:mhajj@stevens.edu

A. Bajaj

School of Mechanical Engineering, Purdue University,

West Lafayette, IN, USA

e-mail: bajaj@purdue.edu as an acknowledgment of his influence on their career and his support. A variety of topics are presented, so that the issue succeeds in providing a sort of updated summary of challenging problems of nonlinear dynamics in mechanics and engineering. This is consistent with the great variety of Nayfeh's interests and contributions, as also highlighted by Rega in the first paper where they are framed within some main stages of development of nonlinear dynamics in the last 40 years. In the following, the technical content of the S.I. is summarized, attempting to frame the different topics within some main macrothemes, pointing out how several of them can be looked at through either a methodological or an application perspective, and always trying to single out Nayfeh's scientific legacy as directly, or more subtly, embedded in many of the addressed themes.

\section{Method of multiple time scales}

The method of multiple time scales (MMS or MTS) and its applications to nonlinear vibration problems are certainly a fundamental part of Nayfeh's legacy. The method is used worldwide to solve weakly nonlinear dynamical problems by thousands of scientists; thus, it is not surprising that also an important number of engineering topics in this S.I. are addressed through the MMS. In some papers, it plays a central role for the analysis of nonlinear oscillations of a mechanical or structural system, with the relevant outcomes being generally complemented and verified via numerical integration of system equations. In other papers, the interest is more widely paid to a variety of 
response aspects, which also include strongly nonlinear dynamics and the relevant techniques, but the MMS is employed to get useful predictions in the weakly nonlinear regime. Single and, mostly, multiple degrees-offreedom (d.o.f.) models of finite- (discrete) or infinite(continuous) dimensional systems are considered. In the latter case, the Galerkin-based discretized formulation of the method in which MTS are applied to a reduced-order model described by ordinary differential equations (ODEs) is generally employed; however, the direct perturbation approach operating on partial differential equations (PDEs) of the infinite-dimensional system is also used, sometimes with comparison purposes. External and parametric resonant excitations are considered, along with the multimode interactions associated with a variety of possible cases of internal resonance, whose effects are analyzed through high-order asymptotic approximations, too. Systems and structures in macro- and micro-/nano-mechanics are dealt with, along with coupled problems. All of the previous alternative aspects have been the object of advanced research by Ali Nayfeh and collaborators, over his long scientific career, with important outcomes in both theoretical and application terms.

Specifically, MTS are used herein to the following purposes.

- To study interactions between self-, parametric and external oscillations in a single-d.o.f. Duffing-type oscillator with two different models (van der Pol or Rayleigh) of self-excitation, and also including a time delay. The method is applied twice, to determine amplitudes of periodic (slow flow) and of quasiperiodic (slow-slow flow) motions (Warminski).

- To predict the nonlinear response of a pendulum cable and describe the influence of the friction and the radius of the support on the change of amplitude of oscillations (Bertrand, Ture Savadkoohi and Lamarque).

- To develop approximate solutions to the modal equations arising from multi-d.o.f. systems with general, non-proportional damping, detuning, and a broad class of cubic nonlinearities, which also highlight the energy-dependent nature of the instantaneous damping (Mathis and Quinn).

- To reproduce experimental data on nonlinear vibrations of suspension bridges via a modified version suitable to study the nonlinear response of struc- tures with damping features depending on natural frequencies of vibrations, named fractional derivative expansion method (Shitikova).

- To highlight variable features of multimodal interaction in a Galerkin-based model of multicablestayed shallow arch bridge, subjected to external and/or parametric excitations and multiple internal resonance (Kang, Guo and Zhu).

- To investigate the influence of multifrequency excitation on the response of an inclined marine riser in conditions of 2:1 and 3:1 internal resonances, with both direct and Galerkin-based formulations, showing how the features of energy exchange and the likelihood of Hopf bifurcations ending up to quasiperiodic and complex motions are affected differently by the two resonance cases (Alfosail and Younis).

- To analyze the dynamic behavior of a twin-well oscillator subjected to an aerodynamic lift force which triggers galloping oscillations, focusing on obtaining an approximate analytical expression for the flow velocity at which steady-state dynamics switches from intra-well to inter-well type. The escape speed is approximated via two different MTS solutions of the governing equation of motion using either harmonic or Jacobi elliptic basis functions (Alhussein and Daqaq).

- To obtain simple analytical expressions for the prediction of spatial shift in vibration phase caused by fluid flow and imperfections of various kinds in a two-mode Galerkin approximation for the flexural vibrations of a fluid-conveying pipe. Analytical approximations are validated by comparing to results of numerical simulation of the full equation of motion, based on a Galerkin expansion with many modes, and for parameter sets of imperfections ranging from "small" (realistic) to "large" (orders of magnitude larger than realistic) (Thomsen and Fuglede).

- To provide a methodology to passively suppress primary, sub- and super-harmonic resonances of a cantilever beam by tuning the inertia nonlinearities such that the response is nearly linear even under strong resonant excitations and various types of nonlinear effects are annihilated (Stanton, Culver and Mann).

- To study stationary resonance regimes of vibrations of a three-d.o.f. system with a limited power supply (non-ideal system) having the Mises girder as 
absorber, via two approximations considering small or large stiffness of the elastic springs of the Mises girder (Mikhlin, Onizhuk and Awrejcewicz).

- To analyze a pair of coupled thermo-optical MEMS oscillators, focusing on the stability features of in-phase and out-of-phase modes (Rand, Zehnder, Shayak and Bhaskar).

- To obtain the relationship between nonlinear velocity feedback gain and controlled response amplitude in self-excited weakly coupled microcantilevers (Nakamura, Yabuno and Yano).

- To analyze simultaneous 1:1 and 2:1 internal resonances among first two symmetric and first antisymmetric modes in an electrothermally tuned and electrostatically actuated micromachined arch resonator, via the direct formulation combined with a Galerkin procedure (Hajjaj, Alfosail, Jaber, Ilyas and Younis).

- To obtain conditions for the existence of bi-stable solutions and an asymptotic estimation of the nonlinear frequency shift which enables spin detection, in the limiting adiabatic model of a continuum mechanics-based resonator for measurement of electron spin via magnetic resonance force microscopy (Hacker and Gottlieb).

- To investigate the nonlinear dynamic response of carbon nanotube (CNT)/polymer nanocomposite beams to primary resonance base excitations, with a generalized Bouc-Wen hysteretic momentcurvature relationship accounting for the hysteresis due to CNT/polymer interfacial frictional sliding, introduced asymptotically via an approach that allows to obtain a discretized model accounting for the piecewise exponential dependence between moment and curvature (Formica and Lacarbonara).

- To obtain an approximate solution of the governing nonlinearly coupled electro-elastic equations of a piezoelectric disk subjected to high-level acoustic excitation, such to form the basis for a nonlinear identification of parameters representing nonlinear piezoelectric coefficients by exploiting the vibrational response of the disk operating in thickness mode under dynamic actuation (Meesala, Hajj and Shahab).

- To derive algebraic approximations of the periodic contact force oscillations bifurcating from stability boundaries in a single-d.o.f. mechanical model of delayed force control with non-collocated force sensor configuration, which turn out to be accept- able in engineering applications for delay parameters within $15 \%$ of their corresponding critical values. This confirms the suitability of nonlinear analysis of delay systems via the MMS, besides the rigorous and more widely used center manifold reduction, as formerly discussed by Nayfeh by comparing the two approaches in the presence of delay parameters (Zhang and Stépan).

\section{Nonlinear dynamics in macro- and micro-/nano- mechanics}

Interest toward nonlinear phenomena in the dynamical behavior of engineering systems encompassed the whole research activity of Ali Nayfeh, moving from the analytical study of nonlinear oscillations of archetypal systems up to the combined analyti$\mathrm{cal} /$ computational/experimental investigations of nonlinear interaction in multimode models of systems and structures, and progressively widening the application fields of interest from macro- to micro-mechanics. In this S.I., these active (and indeed constantly enriched) research lines are represented by a considerable number of scientific topics.

While being generally addressed in the background of specific applications of engineering interest, some of the topics in the previous list of themes addressed via MTS refer to discrete models which can be considered to represent kinds of archetypal situations. This is the case of the parametrically and externally excited Duffing-type oscillator with van der Pol or Rayleigh kind of self-excitation and a time delay aimed at controlling the system response (Warminski); of the pendular cable wrapped around a wheel support, with a special constitutive law subjected to a time-dependent velocity (Bertrand et al.); of a lumped-parameter model of galloping oscillator whose steady-state dynamics switches from intra-well to inter-well type (Alhussein and Daqaq); of simplified third-order models for the dynamics of a microscale oscillator, or of a coupled pair of oscillators, with thermo-optical feedback (Rand et al.); of a model of self-excited weakly coupled microcantilevers with feedback control for proper sensing of a small mass in highly viscous environments (Nakamura et al.); of the single-d.o.f. mechanical models of delayed force control with collocated and noncollocated force sensor configurations, also accounting for large time delays in the feedback loop as possibly occurring in the case of human or remote force control (Zhang and Stépan). 
However, other topics refer more explicitly to nonlinear vibration problems of interest in macro- or micro/nano-mechanics. The former include structural models for the analysis of a suspension bridge or plates in conditions of multiple internal resonance (Shitikova); of a cable-stayed shallow arch bridge (Kang et al.); of an inclined marine riser (Alfosail and Younis); and of a fluid-conveying pipe for applications like Coriolis flow metering (Thomsen and Fuglede). The latter are concerned with the internally resonant dynamics of an electrothermally tuned and electrostatically actuated micromachined arch resonator (Hajjaj et al.); with investigating local and global bifurcations in a continuum mechanics-based resonator for measurement of electron spin via magnetic resonance force microscopy (Hacker and Gottlieb); with the rich dynamic response of different hysteretic nanocomposite beam models, driven by an unusual combination of material and geometric nonlinearities (Formica and Lacarbonara); with the analysis of the acoustic-electro-elastic response of a piezoelectric disk to high-level acoustic excitation, in the context of ultrasound acoustic energy transfer (Meesala et al.).

But other topics of interest in a variety of engineering applications are considered, as well.

Mathis and Quinn deal with the energy transfer in the transient response of a general resonant multi-d.o.f. system due to nonlinearities, focusing on the effect of mode coupling in systems with light generalized linear damping, linear detuning and cubic nonlinearities. 3:1 internal resonance is also considered, dwelling on how energy may more easily transfer from low modes to high modes than vice versa, as commonly observed in experimentation and numerical studies.

Chtouki, Lakrad and Belhaq investigate quasiperiodic bursters and chaotic dynamics in a single-d.o.f. model of shallow arch, subject to slow and fast harmonic motions of one support, and described by a nonlinear QP Mathieu-Duffing equation. Invariant slow manifolds corresponding to the arch undeformed, buckled and unbuckled configurations and ruling the dynamics of the quasiperiodically excited system are computed with the averaging method. Even small amplitudes of high-frequency harmonic excitation are seen to suppress the chaos introduced by low-frequency excitation.

Náprstek and Fischer deal with the dynamics of a heavy ball rolling inside a semi-spherical cavity under horizontal kinematic excitation, considered as a the- oretical setup representative of ball-type tuned mass dampers used in slender engineering structures. The six-d.o.f. system with three non-holonomic constraints is addressed via the Appell-Gibbs approach in the autoparametric resonance zone under uniaxial excitation, and the influence of rolling and spinning dampings at the ball-cavity contact is discussed in the case of biaxial excitation.

Steindl, Edelmann and Plöchl analyze the stability of steady state cornering of a basic nonlinear two-wheel vehicle model with oversteer characteristic and coupled longitudinal and lateral dynamics, described by a full set of equations. Handling properties are discussed, and numerical bifurcation analysis is performed with the steering angle and drive torque as bifurcation parameters. Besides small amplitude limit cycles close to the Hopf bifurcation point, large amplitude relaxation cycles occur where the cyclic motion of the vehicle may be separated into "slow" longitudinal velocityrelated segments, and "fast" yaw and side slip-related segments.

Wiercigroch, Kovacs, Zhong, Costa, Vaziri, M. Kapitaniak and Pavlovskaia present the design evolution and initial experimental results of a novel impact oscillator rig, in which the excitation force is generated electromagnetically and acts directly on the mass, in contrast to most similar rigs where it passes through the structure. This significantly enhances the flexibility of the system control, allowing to follow a particular orbit under parameter changes. Preliminary results demonstrate a wide range of nonlinear responses including periodic orbits, coexistence of attractors and chaotic behavior, along with the potential of the rig for studying fundamental impact phenomena observed in various engineering systems.

Widyan and Harb deal with electrical machines and investigate the dynamical and operational performance of shunt and permanent magnet DC motors powered by lead-acid batteries for water pumping applications. Considering the nonlinearities of all system components, numerical simulations of the complete dynamical model are conducted. The analysis comprises the starting up of the system and the response following successive step changes in the pumping capacities. The variation of the internal resistance of the battery, and its efficiency and state-of-charge at different system running conditions are outlined. 
Some papers deal more explicitly with (multi)modal interaction in the structural dynamics of macroor micro-/nano-systems.

Nonlinear breathing motions and instabilities of a pressure-loaded spherical hyperelastic membrane are addressed by Soares, Amaral, Silva and Gonçalves, via an energy approach in a fully nonlinear elasticity framework. Depending on material parameters, the spherical membrane under static pressure may display a snap-through behavior which has important effects on free and forced nonlinear vibrations, whose features are analyzed in detail.

The paper by Zippo, Barbieri, Iarriccio and Pellicano deals with the non-trivial effects of temperature on the dynamics of shells, aiming to fill a literature gap. Experimental nonlinear dynamics of a cylindrical shell with a top mass under parametric excitation is investigated in a thermally controlled environment, focusing on the range of excitation frequencies close to resonance with the first axisymmetric mode, whose vibration energy is transferred to the shell radial motion.

The classical phenomenon of frequency crossover and the ensuing multiple internally resonant nonlinear vibrations in a micromachined arch resonator are investigated theoretically and experimentally by Hajjaj et al. in a two-part work. Attention is first paid to the linear modal coupling between first symmetric and antisymmetric modes, exploring the influence of electrostatic excitation on mode hybridization, with features which seem to be different from veering and mode localization phenomena. Then, rich and complex dynamics due to the nonlinear coupling among the first two symmetric modes and the first antisymmetric mode are evidenced.

Shoshani, Dykman and Shaw deal with the nonlinear interaction of mutually coupled resonators with significantly different decay rates. They consider a slowly decaying nonlinear (primary) nano-resonator nonlinearly coupled to a relatively fast decaying linear resonator, showing analytically and numerically that the linear and nonlinear characteristics of the former can be altered in a significant manner from the coupling to the latter. This may provide a means of tuning parameters of a resonator to tailor its nonlinear response, that can be useful in NEMS and MEMS applications.

Najar, Ghommem and Abdelkefi present a multifidelity modeling and comparative analysis of two electrically coupled cantilever microbeams. Two modeling approaches are used to analyze the impact of squeezefilm damping on the dynamic behavior, i.e., a fully coupled nonlinear model with the structural and fluidic domains discretized via Galerkin decomposition and differential quadrature method, and a partially coupled model based on approximating the squeezefilm damping force by a nonlinear analytical expression. The latter shows limitations in properly capturing the microsystem dynamics, especially close to pull-in instability and when exciting with AC voltage near resonance.

\section{Reduced-order models}

Although reduced-order models (ROMs) of infinitedimensional systems are employed in many contributions to analyze nonlinear dynamics, two papers are more specifically aimed at developing efficient reduced-order modeling strategies for classes of problems.

Odofin and Epureanu present ROMs for structures with intermittent contacts, obtained through projection onto a basis of normal modes computed by enforcing special boundary conditions at the contact surfaces. Low-order models are used to obtain the forced response by the harmonic balance method. A frequency-adaptive method allows to efficiently estimate the contact area during vibrations and establish the special boundary conditions to be used for reduction. The novel approach is adaptive in that it creates ROMs of different sizes at different frequencies. The method is applied to two test cases to demonstrate its effectiveness and numerical efficiency.

The paper by Imtiaz and Akhtar is concerned with modeling turbulent flows in a ROM framework. A nonlinear closure model based on the dynamical system approach for the Navier-Stokes equations is presented, by considering the flow past a cylinder with turbulent wake. Upon computing proper orthogonal modes and developing a conventional ROM, a closure term analogous to the large-eddy simulation approach is added to accommodate for the deformation tensor term mainly contributing to the computational cost. The model is computationally efficient as compared to existing turbulent ROMs with the same order of accuracy.

\section{Control of vibration and chaos}

In about the last 20 years of his activity, Nayfeh's research activity expanded toward a variety of other themes, also updating some of his previous research interests. Control of vibration, already addressed in the 1980s in the perspective of quenching nonlinear oscilla- 
tions, was certainly one of them. In this S.I., five papers are more specifically devoted to control of vibration and chaos.

Stanton et al. examine the viability of completely passive control approach based on MTS to elicit dynamic cancelation of unwanted nonlinear vibration characteristics. Focusing on a Duffing oscillator augmented by nonlinear inertial forces, specific expressions are obtained for the relationships between inertial and restoring force parameters that can suppress hysteresis, cusp-fold bifurcations, and higher harmonics, across primary, super-harmonic and sub-harmonic resonances, with critical design points obtained in particular for passive control of the latter. Results are mostly relevant to NEMS/MEMS sensing research and technology, as well as vibration-based mechanical energy harvesting or mechanical filtering.

Vestroni and Casini investigate the performance of a Bouc-Wen hysteretic vibration absorber in the mitigation of the steady-state response of a primary elastic structure. Attention is not limited to 1:1 resonance but is extended to different resonances $(2: 1,3: 1)$ where nonlinear dynamic phenomena are more evident. It is shown that the arising of a superabundant mode after a bifurcation around the first frequency leads to the response reduction in the first mode which is beneficial to the primary structure. The delicate problem of the optimal tuning of the hysteretic absorber is also discussed.

Resonance behaviors of a three-d.o.f. system including a source of energy with limited power supply, a linear oscillator, and a Mises girder acting as absorber of elastic vibrations are studied analytically by Mikhlin et al. by considering 1:1 resonance between the motor frequency and the fundamental frequency of either the linear oscillator or the Mises girder. Numerical simulations show that at frequencies near the second resonance it is possible to find regimes of vibration where both an effective absorption of elastic vibrations and the fast outcome from the resonance region are possible.

Tusset, Balthazar, Rocha, Ribeiro and Lenz deal with the control of a MEMS resonator dynamics to a desired periodic orbit via different techniques, state feedback control based on the Lyapunov-Floquet transformation, optimum linear feedback control and statedependent Riccati equation control. Performance of the controllers in terms of minimum time required to achieve a low error, and effects of system parameter uncertainties are investigated. Thermal dependency of the material characteristics is also taken into account by considering damping with fractional order and control sensitivity to fractional derivative variation.

A novel method in the design of controllers to drive general nonlinear systems to desired periodic or quasiperiodic motions is presented by Sharma and Sinha in a paper that was the last one co-authored by Professor Subash Sinha, who sadly passed away on June 10, 2019. The viability of the approach is demonstrated by controlling chaotic systems via a combination of a nonlinear feedforward controller and a linear feedback controller. The gains for the feedback controller are determined by performing the stability analysis of the closed-loop systems that contain periodic or quasiperiodic coefficients, using the Floquet theory in the former case and an approximate technique in the latter case. Three examples of chaotic systems are studied, a forced Duffing oscillator driven to a two-frequency quasiperiodic square wave and a fixed point, a parametrically forced Lorenz system driven to a periodic orbit whose frequency is irrationally related to the parametric excitation frequency, and a Mathieu-Duffing oscillator driven to a two-frequency quasiperiodic motion.

\section{From VIV to energy harvesting}

Aeroelastic systems were certainly among Nayfeh's former research interests, later on resumed and updated in the last part of his scientific activity also in light of novel achievements on vortex-induced vibration (VIV) and energy harvesting within the community of nonlinear dynamicists in mechanics and engineering. Both fields are object of contributions in this S.I.

Mehmood, Abdelkefi, Hajj and Akhtar perform numerical simulations of the flow over a freely oscillating circular cylinder in the cross-flow direction, to investigate the hysteresis phenomenon near the bifurcation points of the synchronization region leading to VIV. The effect of different initial conditions on the extent of the synchronization region identified in literature experiments is analyzed, characterizing the response via nonlinear dynamics tools for different Reynolds numbers. In the synchronous regime, the response is period-1 at the cylinder's natural frequency, whereas in the post-synchronous regime it is period- $n$ with $n>1$. Around the bifurcation points at both ends of the synchronization region, the response depends on the initial input energy.

Blanchard, Bergman and Vakakis deal computationally with the potential of a rotational nonlinear energy 
sink (NES) for use as an energy harvesting device in submarine currents. For a high value of the Reynolds number at which flow past a linearly-sprung cylinder is three-dimensional and fully turbulent, a rotational NES is shown to be capable of harnessing a significant amount of kinetic energy from the motion of the cylinder, while also leading to substantial suppression of its oscillations. The results are of potentially practical significance for VIV suppression and drag reduction in a high-Re bluff-body flow, and lay down the groundwork for designing NESs with a view to enhancing the performance of VIV-induced power generation in marine currents.

While also motivated by offshore engineering, the paper by Mendes, Mazzilli and Ribeiro is actually more specifically concerned with energy harvesting. It investigates the role plaid by the modal asynchronicity of the nonlinear response of a vertical slender-rod mechanical system, with an attached piezoelectric element, subjected to a parametric excitation due to the waveinduced heave motion of a floating buoy on top of the system. Modal asynchronicity, which is a phenomenon connected with localization, entails a superior system performance as far as energy harvesting is concerned.

Aiming at a resonant piezoelectric energy harvesting capable to account for mechanical and dissipative nonlinearities and for the nonlinear process of AC-DC conversion with non-ideal circuit elements, Leadenham and Erturk present a multiphysics harmonic balance framework which, combining mechanical and electrical nonlinear non-ideal effects, succeeds in predicting the DC electrical output in terms of AC mechanical input. A full set of experiments on a cantilevered bimorph piezoelectric energy harvester validates the modeling method and highlights the need to simulate the full nonlinear dynamics with realistic circuit components, in view of real-world applications.

\section{Wave propagation and localization}

Traveling waves was the subject of the last chapter in Nayfeh's seminal book on Nonlinear Oscillations (1979). In about the last 20 years, wave propagation and localization in discrete and continuous systems with some kind of nonlinearity have become themes of major and updated interest. Six papers in this S.I. can be framed in this context.

Mojahed and Vakakis analytically investigate certain aspects of the nonlinear acoustics, especially traveling breathers, of a one-dimensional, semi-infinite, strongly nonlinear lattice, composed of grounded linear oscillators coupled via essentially nonlinear stiffnesses with purely cubic force-displacement characteristics, and subject to an impulsive excitation at its free boundary. In the limit of small energy, the complexificationaveraging method is employed to prove the existence of a nonlinear energy-dependent propagation zone and two attenuation zones in frequency-energy domain of the corresponding infinite lattice. Traveling breathers in the semi-lattice are also investigated, showing that their speed of propagation is energy-dependent, as is the corresponding dispersion relation.

Gzal and Gendelman analyze the nonlinear dynamics of a finite non-homogenous forced-damped chain, which is considered to model helical compression valve springs in an internal combustion engine. The interplay between non-homogenous structure, multifrequency excitation and nonlinearity leads to two qualitatively different states of periodic responses, propagating and edge states, characterized by weak and strong localization at the forced edge, respectively. Analytical solutions conform to numerical simulations, and experimental tests conducted on real valve springs show potential applications of the findings to the design of valve spring, which has important implications on the engine performance, durability and fuel economy.

Kovacic, Zukovic and Radomirovic deal with a localization phenomenon in two groups of bio-inspired, hierarchically organized, oscillatory systems. The intent is to present consistent and simple physics-based models that reflect the essential mechanical structure of an idealized branched tree-like structure, without axial segments and with only lateral segments. Localized modes are seen to appear not only in linear but also in nonlinear systems, under specific conditions which are investigated theoretically for the two groups of models. The results can be used for studying systems with different types of excitations, as well as for appropriate biomimetic applications, especially related to their utilization as vibration absorbers.

Bifurcations of periodic orbits and band zones in a one-dimensional granular array are numerically investigated by Acar and Balachandran. The effects of precompression on periodic orbits of a two-bead system with Hertzian contact between the grains are studied, along with the ensuing changes in periodic wave propagation characteristics of longer granular chains. Changes in the wave propagation band zones are illus- 
trated, by studying vibration transmission at different frequencies to ascertain the band zone limits.

The problem of flexural wave propagation in a beam resting on a unilateral elastic substrate is addressed analytically by Lenci and Clementi. Although the problem is nonlinear, due to the piecewise behavior of the foundation, an exact solution of the governing equations is obtained, with results which are expected to be accurate within the small displacements realm. By considering only physically admissible solutions out of the manifold of mathematical ones, main wave propagation properties are discussed, including the wave velocity as a function of the stiffness of the unilateral foundation.

Abedinnasab and Hussein derive an approximate explicit frequency-versus-wavenumber dispersion relation for finite-strain wave propagation in a slender Euler-Bernoulli beam, by considering both a conventional transverse motion model and an inextensional planar model incorporating coupled axial-transverse motion. For each model, the analytical solution is obtained to $M$ th-order accuracy with the homotopy analysis method and is verified by comparing with results obtained by numerically solving for the roots of the previously derived exact nonlinear dispersion relation. Convergence takes place right away at first-order accuracy for the inextensional planar beam model.

\section{Multibody dynamics}

Two more areas of general importance in mechanics and engineering are represented in this S.I. The first is concerned with the dynamics and optimization of multibody systems, into which three contributions can be framed.

Potosakis, Paraskevopoulos and Natsiavas focus on the dynamics of multibody systems subject to bilateral motion constraints. A new set of equations of motion, appearing first in a strong form and subsequently in an appropriate three-field weak form, is employed. Upon introducing a suitable penalty and a Lagrange multiplier formulation, the equations are eventually cast in an augmented Lagrangian form. This final set of equations is used as a basis for developing a new time integration scheme, whose validity and numerical efficiency are verified by applying it to several challenging examples, ranging from systems with a simple geometry to a complex industrial application involving components with large rigid body rotation.
Pfeiffer dwells on the machine-process combination, seen as two dynamic systems cooperating for the realization of some process. A well-known example is the projection of arbitrary many robot d.o.f. on a given path, resulting in a set of nonlinear equations with one d.o.f. only. The set with the complete number of d.o.f. is used to construct a mobility space helpful to evaluate the overall performance of a machine with respect to some optimal time, force or energy criterion, for any combination of coordinates and constraints. An approach for general machine and process configurations is presented, based on multibody system theory and transformations from the machine to the process side, and vice versa. Examples of a simple double pendulum machine and of a prescribed path to go are given.

Chernousko deals with two- and three-dimensional motions of a body controlled by an internal movable mass in the absence of external forces. Time-optimal 2D trajectories represented by circular arcs are obtained for the case of internal mass small compared to the body mass. The obtained solutions are modified and adapted for the case of an arbitrary ratio of masses. 3D re-orientation of the rigid body is also implemented as a sequence of three suboptimal plane rotations. This general principle of movement can be useful for mobile robots and is also applicable to the attitude control of spacecraft.

\section{Applied dynamical systems}

The second area of general interest is more mathematically oriented and includes papers focusing on methodological and computational aspects of nonlinear dynamics or on complex dynamical systems.

Hamzi and Abed study an extension to nonlinear systems of a recently proposed approach to the concept of modal participation factors. Modal content of the response of dynamic systems is of interest in many application areas (from electric power networks to vibration of structures), with many proposed approaches. For linear time invariant systems, it consists of the eigenmodes and can be studied analytically. For nonlinear systems, possible global oscillations give rise to global oscillatory modes that might not be connected to the eigenmodes of the system's linearization at an equilibrium. The study focuses on local modal analysis of nonlinear autonomous systems, with particular attention to what can be viewed as eigenmodes in the equilibrium neighborhood. 
Maggia, Eisa and Taha show how higher-order averaging can be used to remedy serious technical issues with the direct application of the averaging theorem. Two higher-order averaging methodologies developed independently via different tools and within different communities, i.e., from perturbation theory using Lie transform and chronological calculus, are reconciled. The underpinning concepts behind each approach and a mathematical proof for their equivalence are provided. Two applications of higher-order averaging to mechanical systems are presented: the classical problem of the Kapitza pendulum, and the modern application of flapping dynamics of microair vehicles and/or insects, a topic which Nayfeh also meaningfully contributed to in the last years of his life.

Ahsan, Dankowicz and Sieber generalize a previously conceived continuation-based optimization technique for scalar objective functions on constraint manifolds to cases of periodic and quasiperiodic solutions of delay-differential equations. For both periodic and quasiperiodic dynamics, explicit adjoint conditions taking the form of piecewise-defined, coupled, advanced differential equations are derived via a Lagrange formalism. The methodology is illustrated using the software package $\mathrm{COCO}$ on periodic orbits of both linear and nonlinear delay-differential equations, for which closed-form solutions are not typically available even in the linear case. Optimization on a family of quasiperiodic invariant tori is demonstrated in the unfolding of a Hopf bifurcation with delay and parametric forcing.
Nadini, Bongiorno, Rizzo and Porfiri deal with systems described through time-varying networks of interactions that encapsulate information sharing between individual units over time. These interactions can be classified as reducible or irreducible, the former pertaining to node-specific properties, the latter reflecting dyadic relationships between nodes that form the network backbone. The process of filtering reducible links to detect the backbone network may allow to identify family members and friends in social networks or structures from contact patterns of individuals. A new method for detecting network backbones against time variations in node properties is presented, with analytical insight and numerical evidence of the approach viability for the discovery of network backbones from time series.

We conclude this survey of scientific papers dedicated to the memory of Professor Ali H. Nayfeh by gratefully thanking all of the renowned senior colleagues and younger collaborators who accepted to contribute meaningful research outcomes to this Special Issue. Needless to say that the Editor-in-Chief of Nonlinear Dynamics, Professor Walter Lacarbonara, is also gratefully acknowledged for having taken this due initiative to honor a worldwide recognized scholar and scientist who was also, likely, his most influential mentor.

Publisher's Note Springer Nature remains neutral with regard to jurisdictional claims in published maps and institutional affiliations. 